\section{Chemical bondage}

\section{Geoffrey Wilkinson}

\section{Dictionary of Organometallic}

Compounds, Vols 1,2 and 3 .

Executive editor J. Buckingham.

Chapman \& Hall/Methuen: 1984.

Pp.3,000. £495, $\$ 990$.

Although some organoarsenic compounds were known previously, organometallic chemistry, in which organic molecules or radicals are bound to metallic elements by metal-carbon bonds, really originated with two major discoveries in the nineteenth century. In 1827, in Copenhagen, Zeise discovered the salt $\mathrm{K}\left[\mathrm{PtClC}_{1} \mathrm{H}_{4}\right]$ whose constitution as an ethylene complex, the first of the so-called " $\pi$ complexes", was not fully established until the 1950s. Then in 1845, at a school in Hampshire, Frankland discovered the first true alkyl, diethylzinc. This discovery not only revealed a new area of chemistry but contributed substantially to the concept of valency. The later discovery of magnesium reagents by Grignard opened up even more possibilities as these were easier to handle as synthetic reagents than the flammable zinc compounds. The third major class is the carbon monoxide compounds, discovered by Schutzenberger and by Mond. Although "carbonyls" are not, by strict definition, organometallic, they have metal-to-carbon bonds and carbon monoxide chemistry is now so interlinked with that of the alkyls, aryls, olefins, acetylenes and related or derived compounds that it has become usual to include carbonyls under the general title.

Organometallic chemistry thus includes all manner of compounds, many of which are extremely unusual both in their structure and bonding. During the first half of this century the chemistry was dominated by organocompounds of the main elements - lithium, magnesium, mercury, silicon, phosphorus, arsenic and so on - and by utilization of magnesium and lithium alkyls and aryls in organic synthesis. Transition-metal organometallic chemistry developed only after about 1952 . Carbonyl chemistry proceeded essentially independently, mostly in pre-war Germany through the work of Hieber and his school and of Fischer, Tropsch, Roelen, Reppe and others in industry where the use of transition metal compounds led to the development of new syntheses and to new industrial processes.

Over the past 35 years these two major streams have, to a large extent, merged, and organometallic chemistry today is a major field with its own journals, works of reference and textbooks. The number of papers and patents published annually has risen from a handful to thousands.

This Dictionary provides a list that comprises "those compounds which are in the opinion of the Specialist Editors, most typical, representative, interesting and useful or potentially useful and [presents] their properties and selected bibliography in an orderly and systematically indexed fashion". Quite a mouthful. The elements are treated in alphabetical order from silver (Ag) to zirconium. Each entry includes details of stoichiometry and molecular weight, and of some simple properties such as form, solubility, thermal and air stability, and toxicity, together with a few references to synthesis and spectra. The code-numbered compounds are also given their systematic name which many may find useful; for some of the more complicated compounds, trying to work out the name is often a substantial challenge and far more difficult than in organic chemistry. Structural diagrams are commonly provided and a useful feature is that these are repeated in the summary list at the beginning of each section. The index volume contains full names, molecular formulae and CAS registration numbers.

For workers in organometallic chemisty

\section{Starry night}

\section{Carole Stott}

\section{Maps of the Heavens.}

By George Sergeant Snyder.

André Deutsch, London, Abbeville, New York: 1984. Pp.144. £19.95, \$45.

THE game of joining the dots is not new The Babylonians had such a pastime. They drew pictures by joining dots of light, or rather stars, in the sky. This ancient tradition was followed by Ptolemy whose Almagest (circa AD 150) lists over a thousand stars, grouped into 48 pictures or constellations. Today the sky is divided into an internationally accepted system of $\mathbf{8 8}$ constellations of which the Ptolemaic group forms the core. Many of the very earliest artistic representations are lost forever, and it is only from the advent of the printing press in the sixteenth century that we have examples in abundance. The first printed star maps were Dürer's, of 1516 , and in the 300 years following, artists, mathematicians, cartographers and astronomers have all set down their own interpretations of the dots in the sky.

In Maps of the Heavens, George Snyder, who is in charge of maps and cartographic materials at Sotheby's New York office, looks at the evolution of the iconography of constellations in these more recent maps. The familiar 48 creatures and figures of mythology - Pegasus, Andromeda, Perseus, a lion, a dragon, dogs and bears and the rest, all of which Snyder lists were joined by new figures. The observational work of astronomers such as Tycho Brahe, the revelations of the telescope and voyages to the Southern Hemisphere added new stars ready for forming into constellations. The stars and catalysis, in both academic and industrial laboratories, this mammoth work will be very useful. It may be regarded as the Red Bible to supplement the now wellknown Green Bible, otherwise the ninevolume Comprehensive Organometallic Chemistry published by Pergamon in 1982. The latter has discussions of various sorts in the descriptive text with copious data tables, while the Dictionary is designed to allow rapid access to limited synthetic and structural information. Readers may notice omissions; thus, out of the 145 manganese compounds no simple alkyl, such as $\mathrm{Mn}$ (norbornyl) $)_{4}$ or $\left[\mathrm{Mn}\left(\mathrm{CH}_{2} \mathrm{SiMe}_{3}\right)_{2}\right]_{n}$ is listed. Nevertheless, the editors' choice is pretty extensive and doubtless the promised annual supplements will fill in gaps as well as provide extensions. Expensive though it is, many institutions will have to acquire this work.

Geoffrey Wilkinson is Sir Edward Frankland Professor of Inorganic Chemistry at Imperial College, University of London. became animals, religious figures, political symbols or tools of the sciences and arts. Some are still in use today; others, like La Lande's immortalization of his cat, Felis, or Schiller's replacement of the 12 signs of the zodiac by the 12 apostles, were shortlived.

Snyder's illustrations, which are predominantly from the sixteenth and seventeenth centuries, appear in almost chronological order. Many of them have been chosen for their beauty, not for their importance as stepping stones in the history of celestial cartography; the reader will search in vain for the influential Islamic constellations of Al Sufi, the popular and extensive Uranographia of Bode, and Piccolomini's star atlas of 1540 (which was not only the first printed star atlas but the first printed set of maps of the stars, as distinct from pictures of constellations). Rather, Snyder addresses himself to the colourful pageantry of celestial mapmaking.

Many of the maps betray their date or cultural and religious background through the artist's adoption of a certain artistic tradition. Here, however, the difference between the Eastern- and Western-style constellations is only hinted at as all but a handful of the 75 illustrations are from European countries. The joy of the book is the colourful and realistic photographic reproduction of the maps, which will appeal to a scientific as well as the intended, more popular audience. Each illustration is captivating in itself, while the complementary text, placed adjacent to the map, is reminiscent of an extended and authoritative catalogue entry. Consequently the book can be started and finished at a minimum of $\mathbf{7 5}$ different points. of the National Maritime Museum. 